\title{
On the Energy Efficiency of Massive MIMO with Space-Constrained 2D Antenna Arrays
}

\author{
Sudip Biswas ${ }^{\dagger}$, Christos Masouros*, Tharmalingam Ratnarajah $^{\dagger}$ \\ ${ }^{\dagger}$ Institute for Digital Communications, The University of Edinburgh, King's Buildings, Edinburgh, EH9 3JL, UK \\ ${ }^{*}$ Dept. of Electronic and Electrical Engineering, University College London, Torrington Place, London, WCEI 7JE, UK \\ Email: Sudip.Biswas@ed.ac.uk
}

\begin{abstract}
We examine the deployment of a large multi-user multiple-input multiple-output (MIMO) system and the resulting Energy Efficiency (EE) considering a $2 \mathrm{D}$ rectangular array with increasing antenna elements within a fixed physical space. The resulting increasing mutual coupling and correlation among the base station (BS) antennas are incorporated by deriving a practical mutual coupling matrix which considers coupling among all antenna elements. We also provide a realistic analysis of the energy consumption using a new model, taking into account the circuit power consumption as a function of the number of BS antennas and then present a performance analysis of the system with respect to EE. Our analysis shows that while spectral efficiency increases with increasing number of BS antennas in a massive MIMO system, EE does not increase boundlessly when the increasing number of antennas are to be accommodated within a fixed physical space and the total power consumed is considered to be a function of the BS antennas. Accordingly, analytic expressions for the optimum number of antennas to attain maximum EE are obtained.
\end{abstract}

Index terms- Antenna correlation, mutual coupling, energy efficiency, massive MIMO.

\section{INTRODUCTION}

To meet the ever-increasing demands for the next generation of wireless communication, large scale MIMO systems equipped with very large antenna arrays at the BS serving smaller number of users [1] are introduced that can attain very high spectral efficiencies (SE) [2]. However, due to the limited availability of wireless spectrum, massive MIMO can only be truly exploited by significantly increasing the number of antennas deployed per unit area [4]. A usual practice when deploying antenna elements is to space them by a distance equal to the wavelength of the transmitted frequency or more. One of the constraints towards this end is the limited availability of physical area at the base station. Massively densified antenna deployment is a way out but it leads to two effects- spatial correlation and antenna mutual coupling. The proximity of the antenna elements as signal sources and electrical components causes antenna correlation and coupling respectively [5]. The nulls and the maximum of the radiation pattern of the antennas are shifted owing to the mutual coupling among them [6]. Mutual coupling effects among antenna elements in 1D linear arrays have been studied in [7]- [12]. While [7] and [8] focus on the performance of adaptive arrays when exerted to mutual coupling, [5], [13] and [14] examine the performance of massive MIMO systems with antenna elements affected by mutual coupling.
Energy consumption and power radiation has become a major health and economic hazard over the years [3]. While [21] discusses the electrical power consumptions of power amplifiers, cooling systems and associated circuits installed at a BS, [16] discusses about designing optimal EE massive MIMO systems. Through this paper, we analyse how massive MIMO bounded by a fixed physical space fares to the demands of increasing EE while contributing towards high SE. While most existing works predict over-optimistic performance assuming arrays with unbounded physical space, the more relevant work in [5] considers only linear arrays and a simplified mutual coupling model. In this work, we consider a more realistic 2D planar array bounded by a fixed physical space with an area of about $1 \mathrm{~m}^{2}$ and analytically account for the full mutual coupling model of the array. We reflect on the uplink of such a system affected by antenna correlation and mutual coupling at the BS while keeping the dimensions of the physical spacing of the antenna array in check. We calculate the EE of this system with the help of a new power consumption model incorporating parameters like power consumed by amplifiers, other digital circuits and loss due to mutual coupling.

\section{System Model}

We consider the uplink of a single cell multi-user MIMO arrangement with one BS equipped with a uniform rectangular 2D antenna array in a fixed physical space of area $\Delta$ as shown in Fig. 1. Each row and column of the antenna array consists of $n$ and $m$ dipole antennas respectively with each element separated from the other by a distance $d$ within a row or a column. $M=n \times m$ is the total number of antennas receiving signals from $K$ single-antenna users with $M \gg K$. The users are assumed to transmit their data in the same time-frequency resource with $\lambda$ being the carrier wavelength. It is also assumed that $d<\lambda / 2$, for antenna coupling to significantly impact the performance of the system. Furthermore, the length and breadth of the rectangular array are $\alpha \lambda$ and $\beta \lambda(\alpha, \beta \in \mathbb{N})$ respectively which leads to the following expressions-

$$
\Delta=\alpha \beta \lambda^{2}, \quad d=\frac{\alpha \lambda}{m-1}=\frac{\beta \lambda}{n-1} .
$$

Let $\mathbf{W}$ represent a semi-correlated frequency-flat channel matrix with no line of sight between the BS and the $K$ users which is modeled as $\mathbf{W}=\mathbf{H F}^{\frac{1}{2}} . \mathbf{H} \sim \mathcal{C N}\left(\mathbf{0}, \boldsymbol{\Sigma}_{M} \otimes \mathbf{I}_{K}\right)$ 


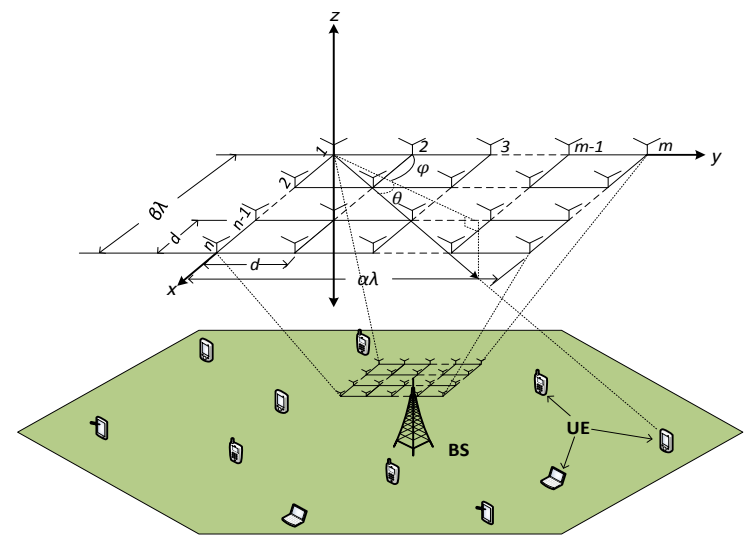

Fig. 1: An illustration of a 2D rectangular array consisting of $M$ dipole antennas serving $K$ single-antenna users located uniformly within the cell diameter in the uplink.

represents the uplink channel where $\boldsymbol{\Sigma}_{M}$ is the BS correlation matrix, $\otimes$ denotes the Kronecker product operator and $\mathbf{F}$ is a $K \times K$ diagonal matrix with $\left[\mathbf{F}_{k k}\right]=\beta_{k} \cdot \sqrt{\beta_{k}}$ models the geometric attenuation and shadow fading which is assumed to be independent over $M$ and constant over several coherence time intervals. This holds true owing to the assumption that $d \ll s_{\text {min }}$, where $s_{\text {min }}$ is the minimum distance between an arbitrary user and the BS and $\beta_{k}$ changes very slowly with time. The $M \times 1$ received vector at the BS can thus be expressed as $\mathbf{y}=\mathbf{W P x}+\mathbf{z}$; where $\mathbf{x} \in \mathbb{C}^{K \times 1}$ is the symbol transmitted by the $K$ users. $\mathbf{P}$ is a $K \times K$ diagonal matrix with $\mathbf{p}=\left[p_{1} \ldots p_{k} \ldots p_{K}\right]$ being the diagonal where the average transmitted power of the users, $p_{i} \geq 0$ for $i=1,2, \ldots, K$ and $\mathbf{z} \sim \mathcal{C N}\left(\mathbf{0}, \mathbf{I}_{M}\right)$ is a vector of additive white Gaussian noise at the BS.

Assuming fading to be correlated only at the BS [18] and incorporating correlation and mutual coupling among the receiving antennas, we model $\mathbf{H}$ as [5]

$$
\mathbf{H}=\left[\begin{array}{lllll}
\mathbf{h}_{1}, & \ldots & \mathbf{h}_{k}, & \ldots & \mathbf{h}_{K}
\end{array}\right],
$$

where $\mathbf{h}_{k}$ is a $M \times 1$ channel vector of the $k$-th user; given as

$$
\mathbf{h}_{k}=\boldsymbol{\Gamma} \mathbf{A}_{k} \mathbf{g}_{k},
$$

where $\boldsymbol{\Gamma} \in \mathbb{C}^{M \times M}$ denotes the mutual coupling matrix, $\mathbf{A}_{k} \in \mathbb{C}^{M \times D}$ denotes the receive steering matrix containing $D$ steering vectors of the receive antenna array with $D$ denoting the number of direction of arrivals (DOAs) and the vector $\mathbf{g}_{k} \sim \mathcal{C N}\left(\mathbf{0}, \mathbf{I}_{D}\right)$ has a dimension of $D \times 1$. Furthermore, $\left[\mathbf{H}^{H} \mathbf{H}\right] \sim \mathcal{C W}_{K}\left(M, \boldsymbol{\Sigma}_{M}\right)$ where $\mathcal{C W}_{K}\left(M, \boldsymbol{\Sigma}_{M}\right)$ denotes a complex Wishart distribution with degrees of freedom $M$, dimension $K$ and covariance $\boldsymbol{\Sigma}_{M}$.

\section{A. Channel correlation at the receiver side}

The steering matrix with respect to the $i$ th DOA can be expressed as

$$
\mathbf{A}_{i}=\mathbf{a}_{c}\left(\phi_{i}, \theta\right) \mathbf{a}_{r}\left(\phi_{i}, \theta\right)^{T}
$$

where $\mathbf{a}_{c}(\theta, \phi) \in \mathbb{C}^{n \times 1}$ and $\mathbf{a}_{r}(\theta, \phi) \in \mathbb{C}^{m \times 1}$ are the column and row array steering vectors respectively given as

$$
\begin{aligned}
& \mathbf{a}_{c}\left(\phi_{i}, \theta\right)=\left[1, e^{j \frac{2 \pi}{\lambda} d \cos \phi_{i} \sin \theta}, \ldots, e^{j \frac{2 \pi}{\lambda} d(n-1) \cos \phi_{i} \sin \theta}\right]^{T}, \\
& \mathbf{a}_{r}\left(\phi_{i}, \theta\right)=\left[1, e^{j \frac{2 \pi}{\lambda} d \sin \phi_{i} \sin \theta}, \ldots, e^{j \frac{2 \pi}{\lambda} d(m-1) \sin \phi_{i} \sin \theta}\right]^{T} .
\end{aligned}
$$

Using the vector valued operator $\operatorname{vec}\{\cdot\}$, which maps a $m \times n$ matrix to a $m n \times 1$ column vector by stacking the columns of the matrix, the array steering matrix may be transformed to a 2-D array steering vector as

$$
\mathbf{a}\left(\phi_{i}, \theta\right)=\operatorname{vec}\left\{\mathbf{A}_{i}\right\} .
$$

The $M \times D$ steering matrix of the array can now be given as

$$
\mathbf{A}=\left[\mathbf{a}\left(\phi_{1}, \theta\right), \ldots, \mathbf{a}\left(\phi_{i}, \theta\right), \ldots, \mathbf{a}\left(\phi_{D}, \theta\right)\right],
$$

where $\mathbf{a}\left(\phi_{i}, \theta\right) \in \mathbb{C}^{M \times 1}$ for $i \in 1,2,3 \ldots, D$. Throughout this paper we consider $d$ to be equidistant and the $D$ DOAs are randomly and independently distributed in an angle spread determined by the azimuth $\phi_{i} \in\left[-\frac{\pi}{2}, \frac{\pi}{2}\right], i=1,2,3 \ldots, D$ and the elevation $\theta \in\left[-\frac{\pi}{2}, \frac{\pi}{2}\right]$. Different degrees of correlation are obtained by varying $\phi$ of the semi-correlated $(D, \phi)$ channel.

\section{B. Mutual Coupling at the receiver side}

The mutual coupling matrix, $\boldsymbol{\Gamma}$ of the array is defined as [6]

$$
\boldsymbol{\Gamma}=\left(Z_{L}+Z_{A}\right)\left(\mathbf{Z}+Z_{L} \mathbf{I}\right)^{-1},
$$

where $Z_{L}$ and $Z_{A}$ denote load and antenna impedance respectively while $\mathbf{Z}$ denotes the mutual impedance matrix.

Let $d_{(i, k)(j, l)}=d \sqrt{(i-j)^{2}+(k-l)^{2}}$ denote the distance between the antenna located at the $i$ th row and $k$ th column and the antenna located at the $j$ th row and $l$ th column of the rectangular array with $i, j \in 1,2, \ldots, n$ and $k, l \in 1,2, \ldots, m$. Then $\mathbf{Z}$ can be constructed as a $M \times M$ matrix as shown in (8) using EMF method [6]. $Z_{(i, k)(j, l)}$ is an element of $\mathbf{Z}$, given as

$$
Z_{(i, k)(j, l)}=R_{(i, k)(j, l)}+j X_{(i, k)(j, l)} .
$$

$R_{(i, k)(j, l)}$ and $X_{(i, k)(j, l)}$ are self-mutual-resistance and selfmutual-reactance between antenna located at the $i$ th row and $k$ th column and the antenna located at the $j$ th row and $l$ th column respectively and given as [6]

$$
\begin{aligned}
& R_{(i, k)(j, l)}=\frac{\sqrt{\mu_{0}}}{4 \pi \sqrt{\epsilon_{0}}}\left[2 C_{i n}\left(u_{0}\right)-C_{i n}\left(u_{1}\right)-C_{i n}\left(u_{2}\right)\right], \\
& X_{(i, k)(j, l)}=\frac{\sqrt{\mu_{0}}}{4 \pi \sqrt{\epsilon_{0}}}\left[2 S_{i n}\left(u_{0}\right)-S_{i n}\left(u_{1}\right)-S_{i n}\left(u_{2}\right)\right],
\end{aligned}
$$

where $\mu_{0}$ and $\epsilon_{0}$ denote the magnetic and electric constants. $u_{0}=2 \pi d_{(i, k)(j, l)}, u_{1}=2 \pi\left(l+\sqrt{d_{(i, k)(j, l)}^{2}+l^{2}}\right)$ and $u_{2}=$ $2 \pi\left(-l+\sqrt{d_{(i, k)(j, l)}^{2}+l^{2}}\right)$ and $C_{i n}($.$) and S_{i n}($.$) are cosine$ and sine integral functions respectively defined as

$C_{i n}(a)=\gamma+\ln (a)+\int_{0}^{a} \frac{\cos t-1}{t} d t, S_{i n}(a)=\int_{0}^{a} \frac{\sin t}{t} d t$, 


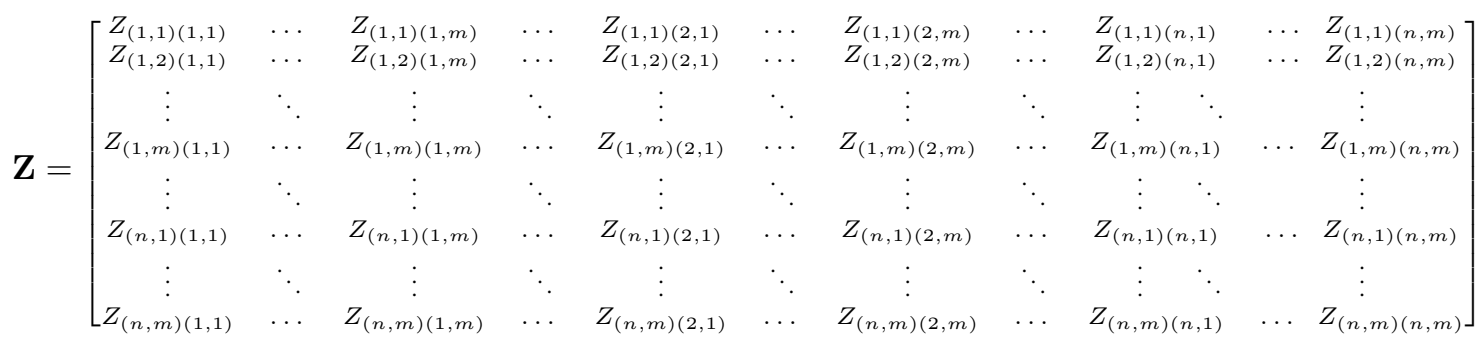

where $\gamma$ is the Euler-Mascheroni constant and $l$ is the length of the dipole antenna. The correlation matrix at the BS can now be given as

$$
\begin{aligned}
\boldsymbol{\Sigma}_{M} & =\mathbb{E}\left\{\mathbf{H} \mathbf{H}^{H}\right\}=\boldsymbol{\Gamma} \mathbf{A} \mathbb{E}\left\{\mathbf{g}_{k} \mathbf{g}_{k}^{H}\right\} \mathbf{A}^{H} \boldsymbol{\Gamma}^{H} \\
& =K \boldsymbol{\Gamma} \mathbf{A} \mathbf{A}^{H} \boldsymbol{\Gamma}^{H}
\end{aligned}
$$

\section{ENERGY EFFICIENCY}

EE of a communication link is the total energy consumption required per bit i.e., the ratio of the sum rate achieved to the total consumed power, and is expressed in bits/joule as [17].

$$
\xi=\frac{R}{p^{P A}+p^{R F}+p^{\text {Coup }}}
$$

where $R$ is the uplink spectral efficiency of the system, $p^{P A}$ and $p^{R F}$ are the power consumed by the power amplifiers and the RF components of the systems respectively and $p^{C o u p}$ is the power loss due to mutual coupling. Hereinafter we consider that every user is guaranteed a minimum fixed rate, $\tilde{R}_{k}$ so as to define $R$ as

$$
R=\sum_{k=1}^{K}\left(\frac{T-K \tau}{T}\right) \tilde{R}_{k}
$$

where $T$ is the length of coherence time interval and $\tau$ is the length of uplink pilots during transmission. To achieve this equal rate condition, we use the approach given in [19] for solving the power control problem in mobile scenarios using Perron's theorem. Accordingly, the power allocated to the $k$ th user can be assigned as

$$
p_{k}=\frac{\gamma_{k}}{q_{k}-\gamma_{k} G_{k i}}
$$

where

$q_{k}=\frac{\left|\mathbf{v}_{k}{ }^{H} \mathbf{w}_{k}\right|^{2}}{\left\|\mathbf{v}_{k}\right\|^{2}}$ and $G_{k i}= \begin{cases}\frac{\left|\mathbf{v}_{k}{ }^{H} \mathbf{w}_{i}\right|^{2}}{\left\|\mathbf{v}_{k}\right\|^{2}}, & \text { for } i \neq k \\ 0, & \text { for } i=k .\end{cases}$

$\gamma_{k}=2^{\tilde{R}_{k}}-1$ is the minimum targeted SINR of the $k$ th user and $\mathbf{V}=\left[\mathbf{v}_{1}, \mathbf{v}_{2}, \ldots, \mathbf{v}_{k} \ldots, \mathbf{v}_{K}\right] \in \mathbb{C}^{M \times K}$ is a linear detector. Since it is much easier to compute the analytic expressions with a $\mathrm{ZF}$ detector, henceforth we consider $\mathrm{ZF}$ detection to compute the analytic results while MRC will be used only for comparison purposes in the simulation results. For $\mathrm{ZF}$ detection, $\mathbf{V}=\mathbf{W}\left(\mathbf{W}^{H} \mathbf{W}\right)^{-1}$. The total transmitted power for $K$ users can now be given as

$$
p_{t}=\mathbb{E}\left\{\mathbf{1}_{(1 \times K)} \mathbf{p}\right\} \text {. }
$$

\section{A. Power Amplifiers}

The average power in watts consumed by the power amplifiers during uplink transmission can be approximated as [17]

$$
p^{P A}=p_{t}(\alpha+1)
$$

where $\alpha=\frac{\zeta}{\eta}-1$ with $\zeta$ being the modulation-dependent peak to average power ratios while $\eta$ is the power amplifier efficiency.

Proposition 1. Let $\bar{p}=f(\gamma)$ be a parameter used to allocate power in order to attain a optimum EE. Hence, considering $Z F$ processing at the $B S$, with no loss of generality, we can define the minimum transmitted rate for all users assuming $M \geq K+1$ as

$$
\tilde{R}=\log _{2}(1+\bar{p}(M-K)) .
$$

Thus the total power consumed by the power amplifiers during one complete cycle of uplink at the BS can be given as

$$
p_{Z F}^{P A}=K \bar{p} \operatorname{tr}\left[\boldsymbol{\Sigma}_{M}^{-1}\right] \frac{\zeta}{\eta}
$$

Proof. From (13) and (17), we have for a $\mathrm{ZF}$ receiver,

$$
p_{k}=\bar{p}(M-K)\left[\left(\mathbf{W}^{H} \mathbf{W}\right)^{-1}\right]_{k k}
$$

Therefore, using the properties of trace of a matrix [20] and from (15) and (16) we have

$$
\begin{aligned}
p_{Z F}^{P A} & =K \bar{p}(M-K) \frac{\zeta}{\eta} \mathbb{E}\left\{\operatorname{tr}\left[\left(\mathbf{W}^{H} \mathbf{W}\right)^{-1}\right]\right\} \\
& =K \bar{p}(M-K) \frac{\zeta}{\eta} \frac{\operatorname{tr}\left[\boldsymbol{\Sigma}_{M}^{-1}\right]}{M-K} \\
& =K \bar{p} \operatorname{tr}\left[\boldsymbol{\Sigma}_{M}^{-1}\right] \frac{\zeta}{\eta}
\end{aligned}
$$

\section{B. RF Chains}

The average power in watts consumed in the RF chains with respect to the $k$ th user can be given as [17]

$$
p_{k}^{R F}=p_{f i x}^{A}+M p^{B S}+K p^{U E}
$$

where $p_{\text {fix }}^{A}$ is the fixed power consumption at the BS dependent on the communication technology, $A \in\{L T E, W I F I\}$, $p^{B S}$ is the power required at the BS to run the circuit components, $p^{U E}$ is the power associated with the user equipments which are defined as follows

$$
p^{B S}=p_{m i x}^{B S}+p_{\text {filt }}^{B S}+p_{A D C}^{B S}+p_{D A C}^{B S}+p_{O S C}^{B S},
$$




$$
p^{U E}=p_{m i x}^{U E}+p_{f i l t}^{U E}+p_{A D C}^{U E}+p_{D A C}^{U E}+p_{O S C}^{U E},
$$

where $p_{\text {mix }}, p_{f i l t}, p_{A D C}, p_{D A C}$ and $p_{O S C}$ denote the power consumed by the mixers, filters, analog-to-digital converters, digital-to-analog converters and local oscillator respectively ${ }^{1}$.

\section{Mutual Coupling}

The mutual coupling effect among antennas in the vicinity of each other as discussed earlier increases the power consumption of the system. The terminal voltage for a particular antenna at the BS can be given as [7]

$$
v_{i}=\sum_{j=1, i \neq j}^{M} i_{j} \Gamma_{i, j}
$$

where, $v_{i}$ denotes the terminal voltage at the $i$ th antenna element due to a unit current in the $j$ th antenna element when the current in all other antenna elements is zero and $\Gamma_{i, j}$ is the total mutual coupling experienced by the $i$ th antenna element due to all other antennas. Furthermore, $\boldsymbol{i}_{B S}=\left[i_{1}, i_{2}, i_{3} \ldots \ldots i_{M}\right]^{T}$, and $\boldsymbol{v}_{B S}=\left[v_{1}, v_{2}, v_{3} \ldots v_{M}\right]^{T}$, where $\boldsymbol{i}_{B S}$ and $\boldsymbol{v}_{B S}$ are vectors of currents and voltages respectively associated with the dipole antennas in the array. The power loss due to coupling based on current maximum now follows as

$$
p^{\text {coup }}=\boldsymbol{v}_{B S}^{T} \boldsymbol{i}_{B S}
$$

The EE of the system now follows from (11-23) as

$$
\xi=\frac{\frac{K}{T}(T-K \tau) \log _{2}(1+\bar{p}(M-K))}{K \bar{p} \operatorname{tr}\left[\boldsymbol{\Sigma}_{M}^{-1}\right] \frac{\zeta}{\eta}+M p^{B S}+K p^{U E}+p^{\text {Coup }}} .
$$

Definition 1. If a local maximum exists in a strictly quasiconcave function, it is also the global maximum [21]. The global optimum can then be obtained by setting the partial derivative of the quasi-concave function to zero.

Proposition 2. Considering ZF processing scheme at BS and diversity loss due to mutual coupling, the optimum number of antennas, $M$ that can be accommodated within a fixed physical space, $\Delta$ which maximizes the EE, $\xi$ can be given by (26) where $W(*)$ is the product logarithm function and $e=2.71828$ is the Euler's number. The rest of the parameters have already been discussed before. Furthermore, it can also be shown that the stationary point $M_{\max }$ is also a global maximum and the EE curve is quasi-concave, which increases for $K+1 \leq M \leq M_{\max }$, attains a global maximum, $M_{\max }$ and then decreases for $M_{\max } \leq M \leq \infty$. Moreover, for $a$ given $M, \bar{p}$ is given by (27).

Proof. We rewrite (25) as

$$
\xi^{Z F}(M)=\frac{\frac{K}{T}(T-K \tau) \log _{2}(1-\bar{p} K+\bar{p} M)}{K \bar{p} \operatorname{tr}\left[\boldsymbol{\Sigma}_{M}^{-1}\right] \frac{\zeta}{\eta}+K p^{U E}+p^{C o u p}+M p^{B S}} .
$$

\footnotetext{
${ }^{1}$ The components considered in this paper may vary from set-ups used in practical scenarios. Any other components used can easily be included in the expressions of $p^{B S}$ and $p^{U E}$ while the ones that are not may be removed.
}

Let $(1-\bar{p} K)=a, \bar{p}=b, K \bar{p} \operatorname{tr}\left[\boldsymbol{\Sigma}_{M}^{-1}\right] \frac{\zeta}{\eta}+K p^{U E}+p^{\text {Coup }}=c$, $p^{B S}=d$ and $\frac{K}{T}(T-K \tau)=f$. Therefore, (28) implies

$$
\xi^{Z F}(M)=\frac{f \log _{2}(a+b M)}{c+d M} .
$$

In order to prove that the objective function, $\xi^{Z F}(M)$ is quasi-concave it is sufficient to prove that the upper contour sets $S_{\psi}=\left\{M \succeq 0 \mid \xi^{Z F}(M) \geq \psi\right\}$ of $\xi^{Z F}(M)$ are convex for any $\psi \in \mathbb{R}[22]$. We investigate the cases when $\psi \leq 0$ and $\psi>0$. When $\psi \leq 0$, the set is empty in the contour $\xi^{Z F}(M)=\psi$. Thus $\xi^{\bar{Z} F}(M)$ is strictly quasi-convex when $\psi \leq 0$. Now when $\psi>0, S_{\psi}$ is equivalent to

$$
\begin{aligned}
& \left\{M \geq 0 \mid \frac{f \log _{2}(a+b M)}{c+d M} \geq \psi\right\} \\
\Longrightarrow \quad & \left\{M \geq 0 \mid c \psi+d M \psi-f \log _{2}(a+b M) \leq 0\right\} .
\end{aligned}
$$

Let $F=c \psi+d M \psi-f \log _{2}(a+b M)$. Now $F$ is strictly convex within the range of $M$ as its Hessian is positive definite. Hence $S_{\psi}$ is strictly convex which concludes that $\xi^{Z F}(M)$ is a quasi-concave function of $M$.

Now using Definition 1, we can say that the local maximum of $\xi^{Z F}$ is also the global maximum. Furthermore as $M \rightarrow \infty$, $\xi^{Z F} \rightarrow 0$. Since $M \not \leq 0$, the local maximum is obtained by calculating the first derivative and setting it to zero as shown below. From (29) we have

$$
\begin{aligned}
\frac{\partial \xi^{Z F}(M)}{\partial M} & =\frac{\partial\left(\frac{f \log _{2}(a+b M)}{c+d M}\right)}{\partial M} \\
& =\frac{f b}{(a+b M)(c+d M) \ln 2}-\frac{f d \ln (a+b M)}{(c+d M)^{2} \ln 2} .
\end{aligned}
$$

Now equating (31) to zero we have

$$
\begin{aligned}
\frac{b(c+d M)}{a+b M} & =d \ln (a+b M) \\
\Longrightarrow \frac{b c-a d}{a+b M} & =d(\ln (a+b M)-1) .
\end{aligned}
$$

Let $\ln (a+b M)-1=x$. Therefore $\exp (x+1)=a+b M$. Thus (32) implies

$$
\begin{aligned}
\frac{b c-a d}{\exp (x+1)} & =d x \\
\Longrightarrow \frac{b c}{d e}-\frac{a}{e} & =x \exp (x) \\
\Longrightarrow x & =W\left(\frac{b c}{d e}-\frac{a}{e}\right),
\end{aligned}
$$

where $W$, known as the product logarithm is the inverse function of $f(W)=W e^{W}$ for any complex number $W$. Substituting $x$ with $\ln (a+b M)-1$ we have

$$
M_{\text {max }}=\frac{\exp \left\{W\left(\frac{b c}{d e}-\frac{a}{e}\right)+1\right\}-a}{b} .
$$

Now replacing $a, b, c, d$ with their equivalent parameters, we have the desired result. Quasi-concavity thus implies that 


$$
\begin{gathered}
M_{\text {max }}=\frac{1}{\bar{p}}\left[\exp \left\{W\left(\frac{\bar{p}\left(K \bar{p} \operatorname{tr}\left[\boldsymbol{\Sigma}_{M}^{-1}\right] \frac{\zeta}{\eta}+K p^{U E}+p^{C o u p}\right)}{p^{B S} e}-\frac{1-\bar{p} K}{e}\right)+1\right\}+\bar{p} K-1\right] \\
\bar{p}_{\text {max }}=\frac{1}{M-K}\left[\exp \left\{W\left(\frac{(M-K)\left(M p^{B S}+K p^{U E}+p^{C o u p}\right)}{K \operatorname{tr}\left[\boldsymbol{\Sigma}_{M}^{-1}\right] \frac{\zeta}{\eta} e}-\frac{1}{e}\right)+1\right\}-1\right]
\end{gathered}
$$

$M_{\max }$ is a global maximum and $\xi^{Z F}$ is increasing for $M<M_{\max }$ and decreasing for $M>M_{\max }$. Thus $M_{\max }$ is the unique optimal $M$ to attain a maximum $\xi^{Z F}$.

Equation (27) can be proved similarly by changing the differentiation variable from $M$ to $\bar{p}$. Accordingly we can parameterize $a, b, c, d$ as $1,(M-K),\left(K p^{U E}+p^{\text {Coup }}+\right.$ $\left.M p^{B S}\right), K \operatorname{tr}\left[\boldsymbol{\Sigma}_{M}^{-1}\right] \frac{\zeta}{\eta}$ respectively. The quasi-concavity of $\xi^{Z F}(\bar{p})$ follows accordingly similar to the proof of (26). Hence, the local maximum will also be the global maximum which can be found by setting the first derivative to zero, i.e.,

$$
\frac{\partial \xi^{Z F}(\bar{p})}{\partial \bar{p}}=0 .
$$

Solving (35) similarly as before, we obtain the desired result. The details are omitted due to space limitations.

\section{RESUlts AND Discussion}

In this section we analyse the behaviour of the proposed model by increasing the number of antennas at the BS while keeping the area of the rectangular array fixed. Typically for a fixed physical spacing, increasing the number of antennas is associated with decreasing the spacing between the antennas, thus increasing mutual coupling which in turn reduces the EE. Therefore, there is a fundamental trade-off between the $\mathrm{EE}$ of the system and the number of antennas at the BS, which will be evident from the simulation results. To obtain a fair comparison, we explore two of the current transmission technologies, namely WIFI and LTE in terms of their fixed transmit power budget.

As stated before, we consider a single hexagonal cell with a diameter of $s_{\max }=3000$ meters which extends from vertex to vertex. The BS is located at the center of the cell with $K=10$ users uniformly distributed in the cell. The minimum distance, $s_{\min }$ between a user and the BS is 50 meters. The large scale fading as described in the system model is defined as $\beta_{k}=$ $\frac{t_{k}}{\left(s_{k} / s_{\min }\right)^{\nu}}$, where $t_{k}$ is the log-normal random variable with a variance $\sigma_{s}^{2}, s_{k}$ is the distance between the $k$ th user and the BS varying anywhere between $s_{\min }$ and $s_{\max }$ and $\nu$ is the pathloss exponent varying from 2 to 4 , with 2 denoting free space propagation and 4 denoting a relatively lossy environment. For our simulations we choose $D=150, \sigma_{s}=10 d B, \nu=$ $3.8, T=196, \tau=10, \eta=0.35$. The length of the dipoles is $0.5 \lambda$ while values of $Z_{A}$ and $Z_{M}$ are considered to be $50 \Omega$. $P_{k}^{\text {mix }}=30.3 \mathrm{~mW}, P_{k}^{\text {filt }}=2.5 \mathrm{~mW}, P_{k}^{A D C}=P_{k}^{D A C}=$ $1.6 \mathrm{~mW}, P_{k}^{o s c}=50 \mathrm{~mW}, P_{\text {fix }}^{W I F I}=25 \mathrm{dBm}$ and $P_{\text {fix }}^{L T E}=$ $43 \mathrm{dBm}$.

We examine the scenario, where the physical spacing of the array is constrained with an area of $1 \mathrm{~m} \times 1 \mathrm{~m}$. Accordingly, we consider three scenarios of fixed physical spaces of $3 \lambda \times 3 \lambda$, $5 \lambda \times 5 \lambda$ and $7 \lambda \times 7 \lambda$ and illustrate the corresponding $\mathrm{EE}$ of the proposed system in Figs. 2, 3 and 4 respectively. The spacing among the antennas varies depending on the number of antennas which in turn affects the mutual coupling matrix. Here we show the achievable EE in a large MIMO system with mutual coupling, considering ZF and MRC detection at the BS. The dashed and continuous lines show the performances of WIFI and LTE systems respectively. On increasing the number of antennas $M$, the spectral efficiency of the system increases. However, $d$ is inversely proportional to $M$ which decreases with increasing $M$; thus also decreasing EE which is evident from the curves. Also to be noted is the shape of the EE curves which are quasi-concave functions of $M$. Furthermore, WIFI systems with low fixed power consumption naturally perform better than LTE systems from EE point of view but the gap in their performance reduces with the increase in the number of antennas. It can also be noted that ZF outperforms MRC in all the three scenarios. The optimal $M$ for $\mathrm{ZF}$ as calculated in section III is also plotted. Our analysis thus shows that number of antennas in the range of 50-120 depending on the array spacing, serving around 10 users can be can be considered to be an optimal solution from EE point of view when mutual coupling is considered among the neighboring antennas.

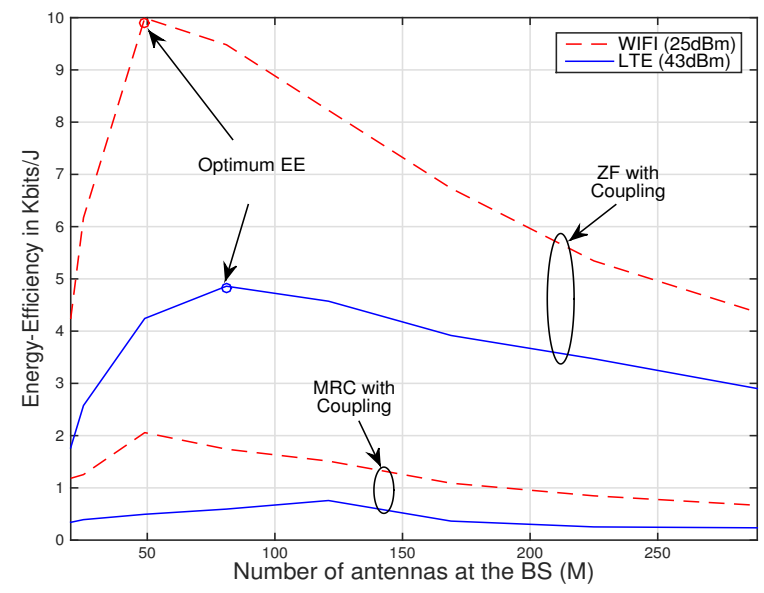

Fig. 2: EE with respect to $M$ considering mutual coupling at the BS for $\alpha=3 \lambda, \beta=3 \lambda$.

\section{Conclusions}

Mutual coupling greatly affects the capacity of a communication system, especially when a large number of antennas are to be rigged within a fixed physical space. In general, 


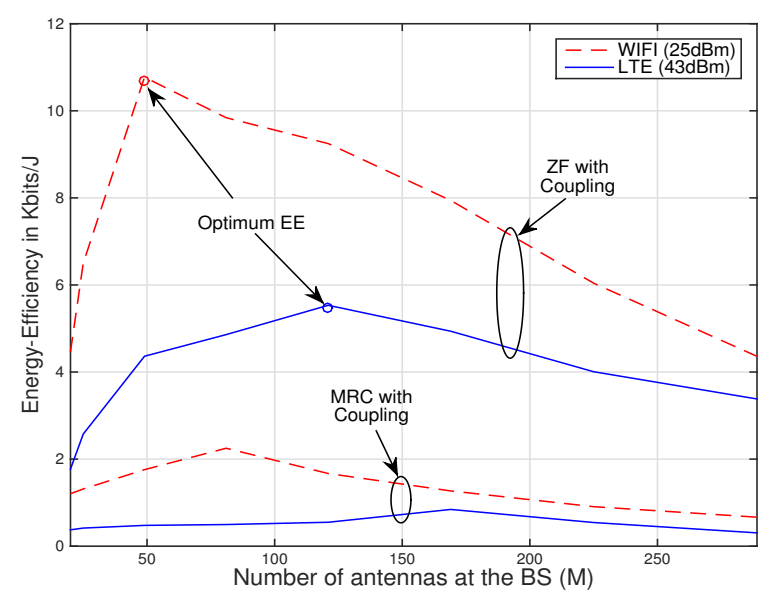

Fig. 3: EE with respect to $M$ considering mutual coupling at the BS for $\alpha=5 \lambda, \beta=5 \lambda$.

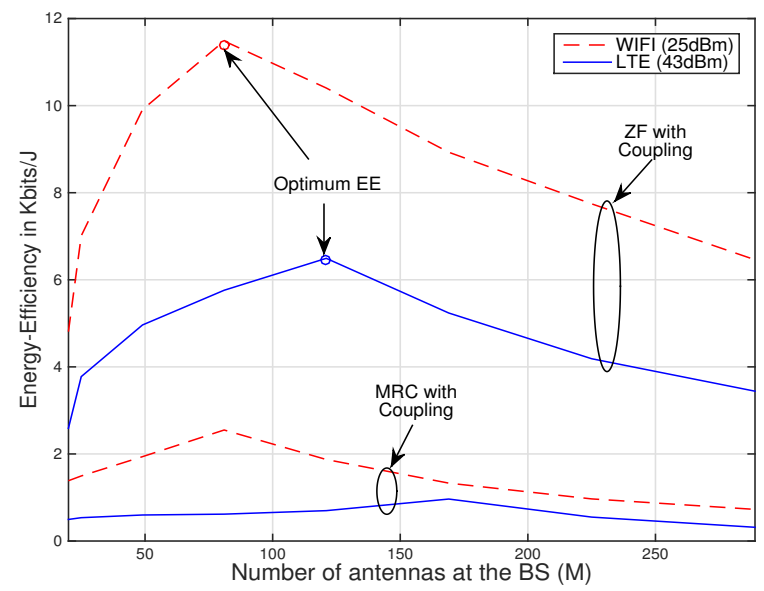

Fig. 4: EE with respect to $M$ considering mutual coupling at the BS for $\alpha=7 \lambda, \beta=7 \lambda$.

massive MIMO systems are considered to achieve high EE with the increase in number of BS antennas which might be misleading when constrained BS physical space and critical antenna spacing are considered. We have given an analysis of the effects of mutual coupling on the EE for a realistic massive MIMO setup. Simulation results show that as the spacing between the antennas is reduced, the coupling among them increases, resulting in a dip in EE performance. Depending on the physical space, an optimum number of antennas is found with the objective of achieving high EE. It is evident that high EE can be obtained but at the cost of reducing $M$ or increasing $\Delta$.

\section{ACKNOWLEDGMENT}

This work was supported by the Seventh Framework Programme for Research of the European Commission under grant number HARP-318489 and the Royal Academy of Engineering, UK.

\section{REFERENCES}

[1] T. L. Marzetta, "Noncooperative cellular wireless with unlimited numbers of base station antennas", IEEE Trans. Wireless Communications, Vol. 9, No. 11, pp. 3590-3600, Nov. 2010.

[2] F. Rusek, D. Persson, B.K. Lau, E.G. Larsson, T. L. Marzetta, O. Edfors, F. Tufvesson "Scaling up MIMO : Opportunities and challenges with very large arrays", IEEE Signal Processing Magazine, Vol. 30, No. 1, pp.40-60, Jan. 2013.

[3] Rhodes and Schwarz, "WLAN 802.11n: From SISO to MIMO", 2010.

[4] J. Hoydis, K. Hosseini, S.T. Brink, and M. Debbah, "Making Smart Use of Excess Antennas: Massive MIMO, Small Cells, and TDD", Bell Labs Technical Journal, Vol. 18, No. 2, pp.5-21, Aug. 2013.

[5] C. Masouros, M. Sellathurai and T. Ratnarajah, "Large-Scale MIMO Transmitters in Fixed Physical Spaces: The effect of transmit Correlation and Mutual Coupling", IEEE Trans. Communications, Vol. 61, No. 7, pp.2794-2804, Jul. 2013.

[6] C. A Balanis, "Antenna Theory: Analysis and Design", 3rd edition.

[7] I. J.Gupta, and A. A.Ksienski, "Effect of Mutual Coupling on the Performance of Adaptive Arrays", IEEE Trans. on Antennas and Propagation, Vol. AP-31, No. 5, Sep. 1983.

[8] Y. Qiaowei, C. Qiang, K. Sawaya, "Performance of adaptive array antenna with arbitrary geometry in the presence of mutual coupling", IEEE Trans. on Antennas and Propagation, Vol.54, No.7, pp.1991-1996, Jul. 2006.

[9] Y.P.Xi, D.G.Fang, Y.X.Sun, Y.L.Chow, "Mutual coupling in a linear dipole array of finite size", IEEE Proc. on Microwaves, Antennas and Propagation, Vol.152, No.5, pp.324,330, Oct. 2005.

[10] T. Svantesson, "The effects of mutual coupling using a linear array of thin dipoles of finite length", In IEEE Proc. on the Ninth Workshop on Statistical Signal and Array Processing, Vol.232, No.235, pp.14-16, Sep. 1998.

[11] R. Janaswamy, "Effect of element mutual coupling on the capacity of fixed length linear arrays", IEEE Antennas and Wireless Propagation Letters, Vol.1, No.1, pp.157-160, 2002.

[12] J. A. G.Malherbe, "Analysis of a linear antenna array including the effects of mutual coupling", IEEE Trans. on Education, Vol.32, No.1, pp.29-34, Feb. 1989.

[13] C. Masouros, C. Jianling, K. Tong, M. Sellathurai, T. Ratnarajah, "Towards massive-MIMO transmitters: On the effects of deploying increasing antennas in fixed physical space", Future Network and Mobile Summit, Vol.1, No.10, pp.3-5 Jul. 2013.

[14] X. Artiga, B. Devillers, J. Perruisseau-Carrier, "Mutual coupling effects in multi-user massive MIMO base stations", In Proc. on the International Symposium Antennas and Propagation, Vol.1, No.2, pp. 8-14 Jul. 2012.

[15] A. Fehske, G. Fettweis, J. Malmodin, and G. Biczok, "The global footprint of mobile communications: The ecological and economic perspective", IEEE Communications Magazine, Vol.49, No.8, pp.55-62, Aug. 2011.

[16] E. Bjornson, L. Sanguinetti, J. Hoydis and M. Debbah, "Optimal Design of Energy-Efficient Multi-User MIMO Systems: Is Massive MIMO the Answer?", available online at: http://arxiv.org/abs/1403.6150.

[17] S. Chui, A. J.Goldsmith and A. Bahai, "Energy-Efficiency of MIMO and Cooperative MIMO Techniques in Sensor Networks", IEEE journal on selected areas in Communications, Vol. 22, No. 6, Aug 2004.

[18] T. Ratnarajah and R. Vaillancourt, "Quadratic Forms on Complex Random Matrices and Multiple-Antenna Systems", IEEE Trans. on Information Theory, Vol. 51, No. 8, pp. 2979-2984, Aug. 2005.

[19] S. Pillai, T. Suel, and S. Cha, "The Perron-Frobenius theorem: some of its applications", IEEE Signal Process. Mag., Vol. 22, No. 2, pp. 62-75, Mar. 2005.

[20] D. K.Nagar, and A. K.Gupta, "Expectations of functions of complex Wishart Matrix", Springer Science+Business Media, B.V., Vol. 113, No. 3, pp. 265-288, Mar. 2011

[21] E. Wolfstetter, Topics in Microeconomics: Industrial Organization, Auctions and Incentives, Cambridge University Press, 1999.

[22] S. Boyd and L. Vandenberghe, Convex Optimization, Cambridge University Press, 2004. 